\title{
Adaptive Digital Beam Forming using LMS Algorithm
}

\author{
B. S. Reddy, Dr. A. S. Bhalchandra, Dr. V. R. Ratnaparkhe \\ (Government collage of engineering, Aurangabad, India)
}

\begin{abstract}
There is a need of Phased Array Antennas in applications such as wireless communication, RADAR systems. The radiation pattern for this type of antenna has to be narrower sharper in one direction and should have nulls in other directions. In order to achieve this, phased array antennas are designed in such a way so as to steer the beam in required direction automatically. One of the techniques described in this paper is LMS algorithm. Nested LMS algorithms are used to optimize the radiation pattern. By varying the step-size, optimizing weights radiation patterns are observed. Change of direction of desired signal is also considered. It is observed that Gain is maximum in desired direction and nulls are created in the direction of noise signals.
\end{abstract}

Keywords: Adaptive algorithm, beam forming, least mean square algorithm, variable step-size.

\section{Introduction}

Basically, an antenna is a device used to transmit or receive the electromagnetic waves [1]. The Directivity or Gain of an isotropic antenna is equal in all directions as shown in fig.1. Generally such antennas are used for broadcasting (transmitter) applications. But for many applications the maximum gain (lobes) is desired in particular direction and attenuation (nulls) is desired in other direction.

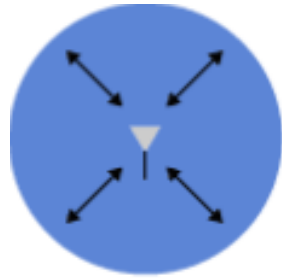

Fig.1: Isotropic antenna

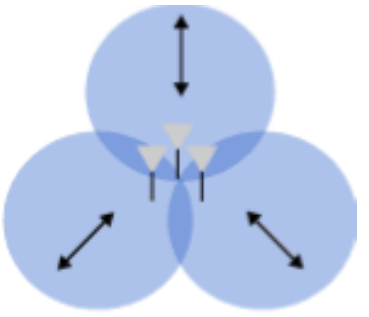

Fig 2: Phased array antenna

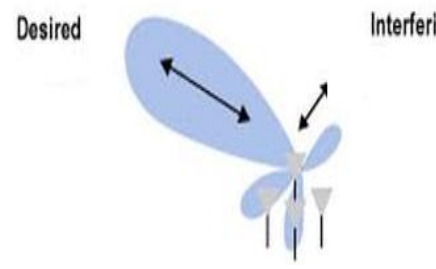

Fig 3: Beam former antenna

Phased array antennas are used to fulfil this requirement. Array of antennas is used to produce maximum Gain/Directivity at particular direction and minimum Gain/Directivity in rest of the directions in fig.2. By controlling (i) excitation of array elements (ii) phase difference of excitation between the adjacent array elements (iii) separation distance between the elements, desired radiation pattern can be achieved. The antenna's radiation pattern describes areas of Gain (lobes) or Attenuation (nulls) for the signals [2].

In some applications, receiver should know the direction of arrival of the signal from the transmitter and accordingly the receiver should be able to steer the beam and acquire maximum signal in specified direction. This adjustment of radiation pattern towards transmitter is called as 'Beamforming'. Changing distance between the antenna elements is not practical solution. Hence either by changing the amplitude of excitation or by changing the phase of excitation steering of beam is achieved in fig. 3 .

To achieve the maximum gain/directivity, phased array antenna elements are connected to the digital signal processor to steer the beam automatically [2]. The process of getting the required maximum directivity in desired direction by using digital signal processing techniques is called as 'digital beamforming'. The process of determining the complex weights and controlling output to get the specified direction is called as 'adaptive digital beam forming'. To determine the complex weights, many adaptive filter techniques are used, which forms the major part of adaptive digital beamforming technology [3].

One of the basic and most important filtering techniques is LMS (least mean square) algorithm. This is used because of its simplicity, small calculations and higher stability [4].

The main problem of adaptive technique is to find the complex weights. There are several ways to produce this weights and inturn produce optimized radiation pattern with minimum side lobes [],[]. This technique reduces beamwidth and improves directivity.

Simple LMS algorithm is modified to optimize the array pattern. This is achieved by changing the gradient or by changing the step-size [4-8]. Variable step-size is also utilized which improves the convergence rate in turn improves the directivity. In mobile communication, receiver should able to receive multiple user 
signals, therefore instead of using constant step-size multiple step size are used [5]. Another way is to use concept of subarray to increase the computational efficiency [9].

In this paper, Adaptive digital beam forming is achieved by using LMS algorithm with variable stepsize. A step-size controls the convergence rate and also determines the mean square error. Convergence rate is inversely proportional to step-size, large step-size leads to fast convergence but results in increase in error. The proposed work, first starts by using LMS algorithm with fixedstep-size. The minimum error is calculated and corresponding weights are selected for next LMS algorithm. Now, by initially fixing these weights, step-size is varied from 0 to 1 , for each step-size value, min error is calculated and corresponding weights are taken for next cycle. Finally radiation pattern is plotted for optimized weights and observed weather the directivity is high in the desired direction.

\section{Digital Beam Forming}

Beam forming is defined as the combination of radio signals from a set of non-directional antennas to simulate one antenna with directional properties. Here, both amplitude and phase of each antenna element are controlled [10]. One of the advantage of this type of control is, it can adjust side lobe levels and steer nulls when compared to phase control alone.

The complex weights are defined as the combination of relative amplitude $a_{k}$ and phase shift $\theta_{k}$ for each antenna and is represented by complex constant $w_{k}$ (for $k^{\text {th }}$ antenna element).In digital beam forming, the operations of phase shifting and amplitude scaling for each antenna element and summation for receiving are done digitally. The receiver beam former model is as shown in fig 4 .

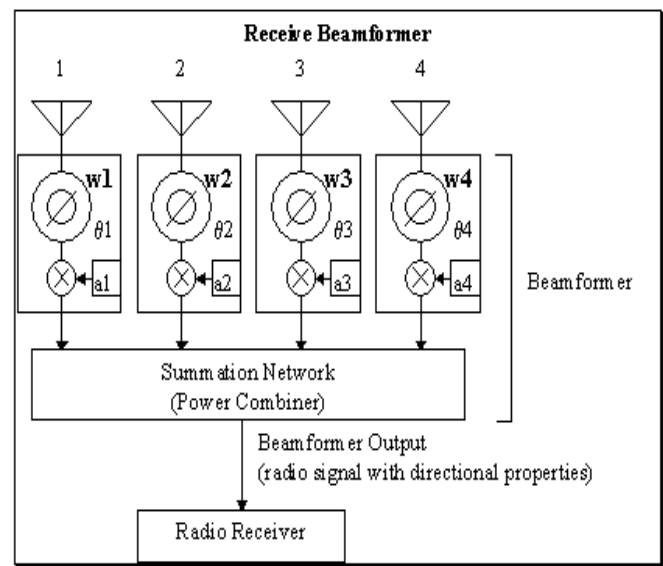

Fig.4: receiver beam former

To make the input signal digital, A/D converters are used. As input signals are radio waves whose frequencies are too high to digitize. Therefore this radio signals are passed through 'RF translator' to shift the frequency from higher frequencies to lower frequencies. After that the signal is digitized. Once the signal is digitized they are passed to 'digital down converters', produces a 'quadrature' base band output at a low sample rate as shown in fig.5.

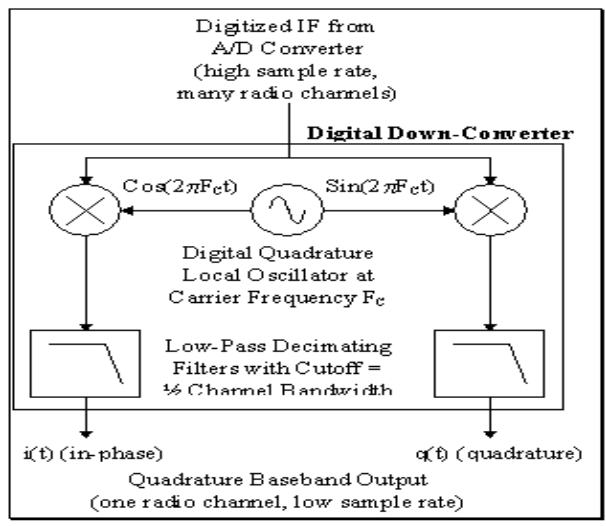

Fig.5: Digital down converter output 
The quadrature baseband signal can be represented as

$\mathrm{s}(\mathrm{t})=\mathrm{x}(\mathrm{t})+\mathrm{jy}(\mathrm{t})$

$s(t)$ is the complex base band signal

$\mathrm{x}(\mathrm{t})=\mathrm{i}(\mathrm{t})$ is the real part

$\mathrm{y}(\mathrm{t})=-\mathrm{q}(\mathrm{t})$ is the imaginary part

$\mathrm{j}$ is $\sqrt{(-1)}$

These complex base band signals are multiplied by the complex weights

$w_{k}=a_{k} e^{j \sin \left(\theta_{k}\right)}$

$w_{k}=a_{k} \cos \left(\theta_{k}\right)+j a_{k} \sin \left(\theta_{k}\right)$

$w_{k}$ - Complex weight for the $k^{\text {th }}$ antenna element

$a_{k}$ - Relative amplitude of the weight

$\theta_{k}$ - Phase shift of the weight

For each antenna element the complex multiplication is done:

$s_{k}(t) w_{k}=a_{k}\left\{\left[X_{k}(t) \cos \left(\theta_{k}\right)-Y_{k}(t) \sin \left(\theta_{k}\right)\right]+j\left[X_{k}(t) \sin \left(\theta_{k}\right)+Y_{k}(t) \cos \left(\theta_{k}\right)\right]\right\}$ As shown in fig 6 .

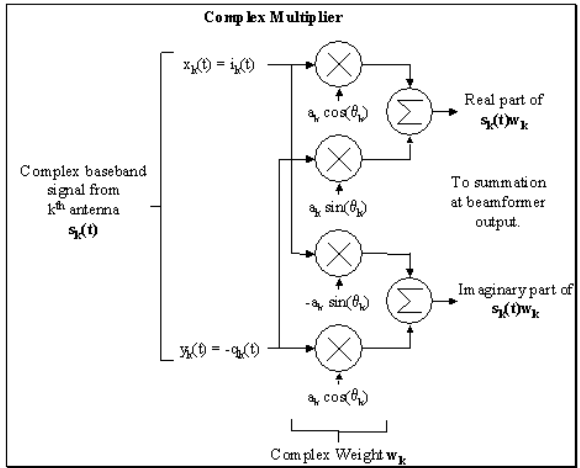

Fig.6: complex weight multiplication

filter. LMS algorithm is based on gradient descent method, which makes consecutive corrections to the weight vector in the direction of negative gradient vector which finally leads to the minimum mean square error.

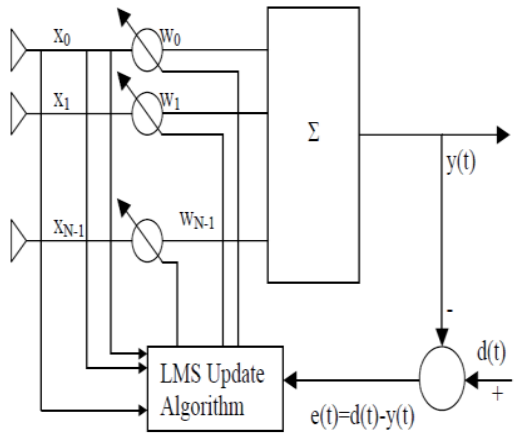

Fig.7: LMS Adaptive Beam Forming Network

$\mathrm{s}(\mathrm{t})$ - Desired signal arriving at angle $\theta_{0}$.

$u_{i}(t)$ - interfering signals at angle $\theta_{i}$.

$\mathrm{n}(\mathrm{t})$ - noise.

$a\left(\theta_{0}\right) \& a\left(\theta_{i}\right)$ - steering vector.

Let, all signals are represented by their sample values. From steepest descent method, the weight equation is represented as

$$
w(n+1)=w(n)+\frac{1}{2} \mu\left[-\nabla\left(E\left\{e^{2}(n)\right\}\right)\right]
$$

$\mu$ - Stepsize (controls the convergence characteristics of LMS algorithm)

$e^{2}(n)$ - Mean square error between beamformer output $\mathrm{y}(\mathrm{n})$ and the reference signal.

$e^{2}(n)=\left[d^{*}(n)-w^{h} x(n)\right]^{2}$

$\nabla\left(E\left\{e^{2}(n)\right\}\right)-$ Gradient vector

$\nabla_{w}\left(E\left\{e^{2}(n)\right\}\right)=-2 r+2 R w(n)$ 
Here ' $r$ ' and ' $R$ ' values are simplified by using the instantaneous values of covariance matrices and is given by $R(n)=x(n) x^{h}(n)$

$r(n)=d^{*}(n) x(n)$

Therefore the weights update equation is

$w(n+1)=w(n)+\mu x(n)\left[d^{*}(n)-x^{h}(n) w(n)\right]$

$w(n+1)=w(n)+\mu x(n) e^{*}(n)$

Where,

$e(n)=d^{*}(n)-y(n)$

$$
y(n)=w^{h} x(n)
$$

The LMS algorithm is initiated with some initial weights, to normally converge and stay stable $\mu$ value should be $0<\mu<1 / \lambda_{\max }$

\section{Experimental Analysis}

In this paper, the adaptive digital beam forming algorithm was developed by means of simulation software called 'Matlab'. The simulation is performed with a linear array antenna and the number of element taken are $4, \mathrm{~d}=\frac{\lambda}{2}, \mathrm{k}=\frac{2 \pi}{\lambda}$ are fixed.

The LMS algorithm contains three steps in each recursion:

1. Computation of the processed signal with the current set of weights.

2. Generation of the error between the processed signal and the desired signal.

3. Adjustment of the weights with the new error information by the gradient method.

The input at each element of array antenna consists of sum ofinput signal which is taken as complex MSK signal in some desired direction $\theta_{d}$, introduced two interrupts in the direction $\theta_{n 1}$ and $\theta_{n 2}$ and introduced random noise signal $\mathrm{n}$. the above signals are shown in fig 7.

The proposed work is divided in following steps

1. Initially simple LMS algorithm by keeping initial weights zeroes and with fixed step-size. Minimum error is calculated and the corresponding weights are fixed and are used as initial weights in case 2 and case 3.

2. In second step, LMS algorithm is developed by setting initial weights derived from case 1 and step-size is varied in proper steps. Here sample by sample optimization is done to get the desired signal.

3. Finally, Performed LMS algorithm for entire samples to get the direction of the desired signal by varying step-size and by setting initial weights.

The following are the observations

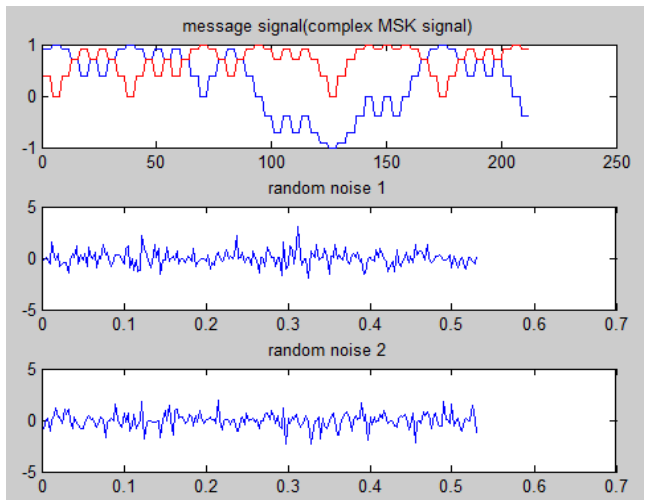

Fig.8: Input signals

$\mathrm{R}$ is produced because error minimum means the output of the beam former is equals to the desired signal. Therefore we have selected the weights corresponding to the minimum error. 

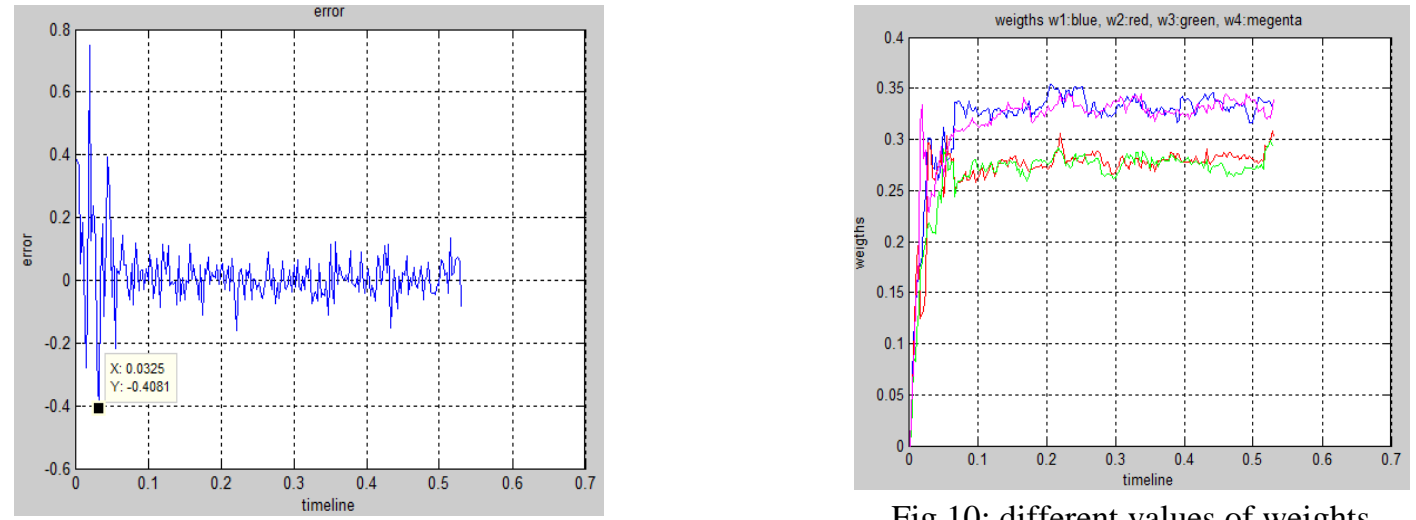

Fig.10: different values of weights

\begin{tabular}{|c|c|c|c|c|c|}
\hline$\theta_{d}$ & Min(e) & W1 & W2 & W3 & W4 \\
\hline 10 & -0.9413 & 0.034 & 0.317 & 0.221 & -0.119 \\
& & $+0.012 \mathrm{i}$ & $-0.304 \mathrm{i}$ & $-0.227 \mathrm{i}$ & $-0.117 \mathrm{i}$ \\
\hline 50 & -0.5166 & 0.168 & -0.202 & 0.049 & 0.326 \\
& & $-0.229 \mathrm{i}$ & $-0.171 \mathrm{i}$ & $+0.274 \mathrm{i}$ & $-0.005 \mathrm{i}$ \\
\hline 90 & -1.0741 & 0.267 & -0.099 & 0.162 & -0.317 \\
& & $-0.126 \mathrm{i}$ & $+0.091 \mathrm{i}$ & $+0.029 \mathrm{i}$ & $-0.011 \mathrm{i}$ \\
\hline 150 & -0.1829 & 0.099 & 0.023 & -0.087 & -0.133 \\
& & $-0.099 \mathrm{i}$ & $-0.181 \mathrm{i}$ & $+0.062 \mathrm{i}$ & $+0.195 \mathrm{i}$ \\
\hline 170 & -0.2382 & 0.167 & 0.202 & 0.097 & -0.046 \\
& & $-0.055 \mathrm{i}$ & $-0.081 \mathrm{i}$ & $-0.171 \mathrm{i}$ & $-0.188 \mathrm{i}$ \\
\hline 340 & -0.3766 & 0.113 & 0.099 & -0.083 & -0.152 \\
& & $-0.109 \mathrm{i}$ & $+0.139 \mathrm{i}$ & $+0.121 \mathrm{i}$ & $-0.078 \mathrm{i}$ \\
\hline
\end{tabular}

Table 1: list of optimized weights with minimized error

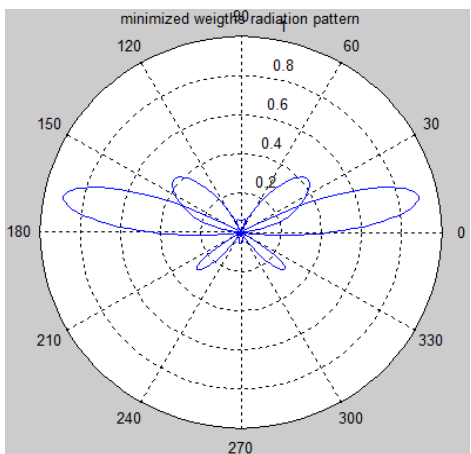

Fig: main lobe at $\theta_{d}=10$, nulls at $\theta_{n 1}=$ $-45, \theta_{n 2}=80$

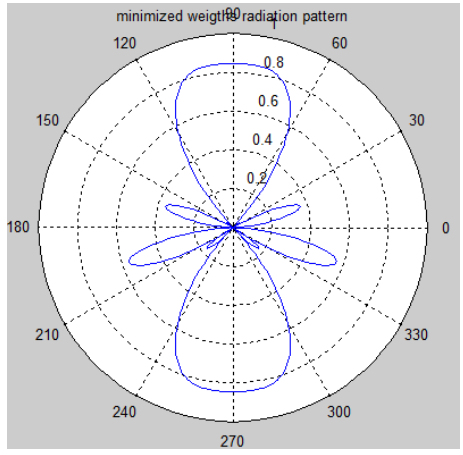

Fig: main lobe at $\theta_{d}=90$, nulls at $\theta_{n 1}=$ $-45, \theta_{n 2}=80$

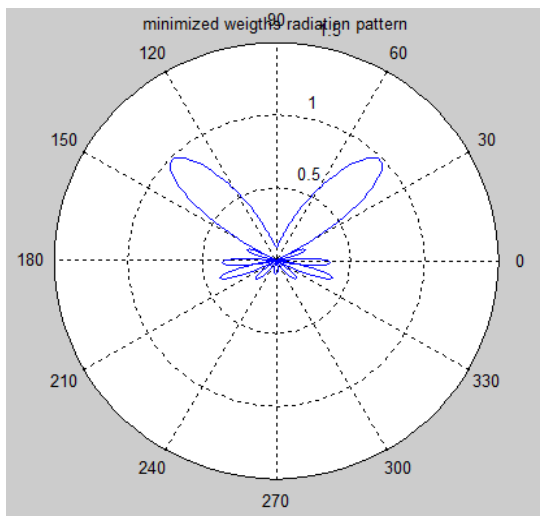

Fig: main lobe at $\theta_{d}=50$, nulls at $\theta_{n 1}=$ $-45, \theta_{n 2}=80$

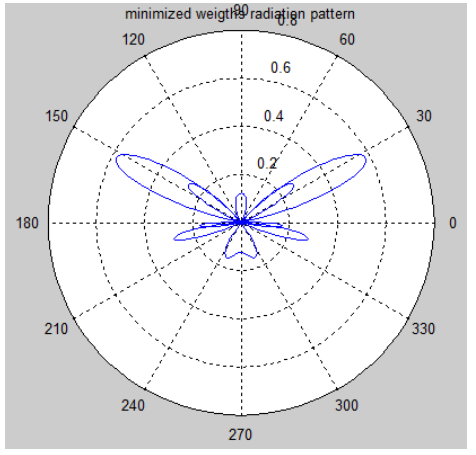

Fig: main lobe at $\theta_{d}=150$, nulls at $\theta_{n 1}=$ $-45, \theta_{n 2}=80$ 


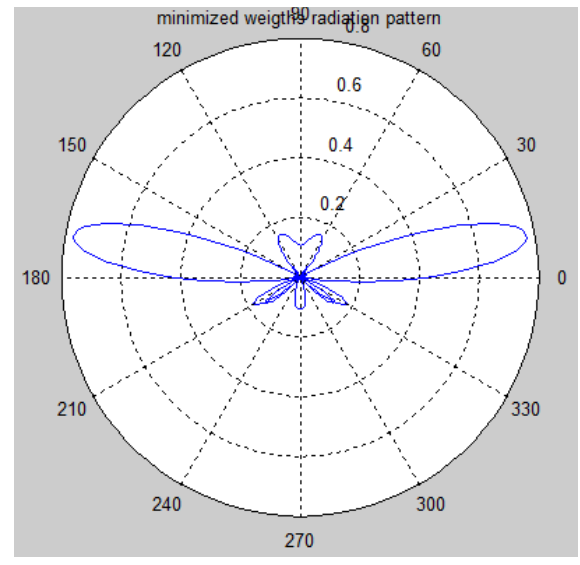

Fig: main lobe at $\theta_{d}=170$, nulls at $\theta_{n 1}=$ $-45, \theta_{n 2}=80$

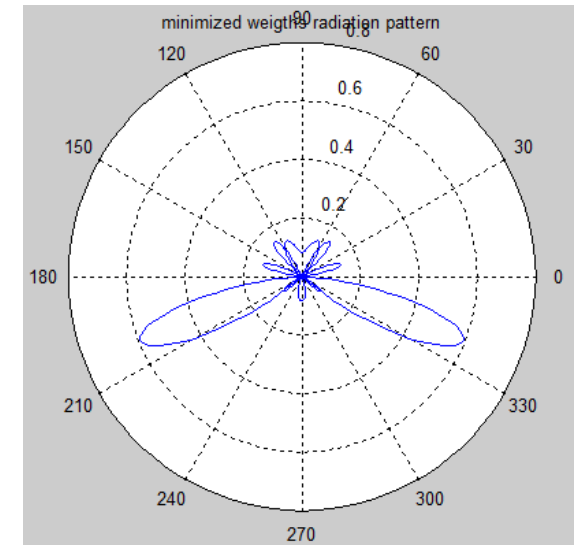

Fig: main lobe at $\theta_{d}=340$, nulls at $\theta_{n 1}=$

$$
-45, \theta_{n 2}=80
$$

The above graphs shows that maximum lobe is observed in the desired direction which is given as an input, also minimum lobe or nulls are observed in noise directions. The LMS algorithm fixes the weights such that maximum directivity is achieved in given input direction.

\section{Conclusion}

All the results have been achieved by using MATLAB 2012 software. Radiation patterns have been theoretically calculated and compared with practical results. The following conclusions have been made depending upon the result obtained.

1. The paper has proposed simple but yet so effective adaptive digital beam forming algorithm called LMS algorithm.

2. Instead of using basic LMS algorithm, some modifications have been done. LMS algorithm is used in three stages. First to find the minimum error at fixed step-size and found the weights. Secondly varied the step-size executed the algorithm for sample by sample beam former output and lastly by varying the step-size for entire sample calculated the minimum error and respective weights are produced. The final weights are used to draw the radiation pattern.

3. From the radiation pattern, observed that whether the direction of desired signal is achieved.

4. The direction of the desired signal slightly differs due to the presence of noise but maximum signal can be retrieved.

5. Hence by applying nested LMS algorithm performance can beincrease.

\section{References}

[1] Balanis, Antenna Theory: analysis and design, (New Jersey: John Wiley \& Sons, C.A (2005)).

[2] SumitVerma\& Arvind Pathak, Digital Beam Forming using RLS-QRD algorithm, International Journal of Engineering Research \& Technology (IJERT), ISSN:2278-0181, Vol.1, Issue 2,July 2012.

[3] Susmita Das, Smart Antenna Design for Wireless Communication using Adaptive Beam-forming Approach.

[4] Maobing Hu, Wei Tang, Canhui Cai, A New Variable Step-Size LMS Adaptive Filtering Algorithm for Beam forming Technology.

[5] Byung Goo Choi, Yong Wan Park, Jeong Hee Choi, The Adaptive Least Mean Square Algorithm Using Several Step Size for Multiuser Detection, 0-7803-6507-0/00, IEEE 2000.

[6] S.C.Upadhyay and P.M.Mainkar, Adaptive Array Beam forming using LMS Algorithm, International Journal of Engineering Research \& Technology (IJERT), ISSN:2278-0181, Vol.2, Issue 1, January- 2013.

[7] LAL C. GODARA, Improved LMS Algorithm for Adaptive Beam forming, IEEE TRANSACTION ON ANTENNAS AND PROPAGATION, VOL. 38, NO. 10, OCTOBER 1990.

[8] Wei Shao, Yujiang Yu, Zuping Qian, An Effective Variable Step Size CS-LMS Algorithm for Adaptive Beam forming, $20102^{\text {nd }}$ International Conference on Signal Processing Systems (ICSPS).

[9] Fei HUANG, Wei Xing SHENG, Bo Yu QI, Wei WANG, A New Fast Adaptive Beam forming Method Based on LMS Algorithm,0-7803-9433-X/05, IEEE 2005.

[10] Toby Haynes, “A Primer on Digital Beam forming”.

[11] Reza SeifiMajdar, Mohammad Eshghi, A New Variable Step-Size Normalized PBS_LMS Algorithm, 978-1-4577-2059-8/11, IEEE 2011. 